\title{
Effects of oral implants with miR-122-modified cell sheets on rat bone marrow mesenchymal stem cells
}

\author{
DAN SHAO, CHUNFANG WANG, YAPING SUN and LEI CUI \\ Department of Stomatology, The First People's Hospital of Qingdao Economic and \\ Technological Development Zone, Qingdao, Shandong 266555, P.R. China
}

Received May 2, 2017; Accepted September 22, 2017

DOI: $10.3892 / \mathrm{mmr} .2017 .8094$

\begin{abstract}
The aim of the present study was to regulate the transformation of bone marrow mesenchymal stem cells (BMMSCs) to osteoblasts to promote bone formation and osseointegration surrounding oral implants. BMMSCs were cultured using the whole bone marrow adherence method. Cell surface markers were detected by flow cytometry, and multi-lineage differentiation potential was detected by osteogenic and adipogenic tests. miR-122-modified cell sheets were prepared by non-viral transfection and complexed with micro-arc titanium oxide implants to construct a gene-modified tissue-engineered implant, with its surface morphology observed by scanning electron microscopy (SEM). In vitro osteogenic activity of the implant was determined by alkaline phosphatase (ALP), Sirius Red, alizarin red staining, polymerase chain reaction and western blot analysis. The BMMSCs were spindle- or triangular-shaped. Surface markers, cluster of differentiation 29 (CD29), CD90 and CD105 were positively expressed, whereas blood cell markers CD34, CD45 and CD31 were negatively expressed. Osteogenic staining exhibited deposition of calcified nodules, while adipogenic staining demonstrated the formation of lipid droplets. miR-122 modification significantly enhanced the in vitro osteogenic activity of the sheets. On day 3 of osteogenic induction, runt-related transcription factor 2, osterix, osteocalcin, collagen I, ALP and bone morphogenetic protein 2 expression levels of the experimental group were 2.0, 3.1, 4.6, 3.2, 10.5 and 4.5 times those of the blank control group, respectively. SEM imaging of the modified sheet demonstrated close adhesion and fitting between abundant cellular and extracellular matrices, and the porous surface of the implant. In vitro osteogenesis of the complex was promoted and accelerated. Thus, miR-122
\end{abstract}

Correspondence to: Professor Yaping Sun or Professor Lei Cui, Department of Stomatology, The First People's Hospital of Qingdao Economic and Technological Development Zone, 9 Huangpujiang Road, Qingdao, Shandong 266555, P.R. China

E-mail: 1329244236@we.com

E-mail: cuileifph@126.com

Key words: cell sheet, bone marrow mesenchymal stem cell, gene transfection, microRNA, implant effectively promoted osteogenic differentiation of the BMMSC sheet. Therefore, it is feasible to construct gene-modified tissue-engineered implants by complexing miR-122-modified sheets with micro-arc titanium oxide implants.

\section{Introduction}

Cell sheet technology, as the main research direction of modern regenerative medicine, refers to the continuous culture of high-density inoculated cells in vitro for multi-layer growth to form a membranous structure that is rich in cellular and extracellular matrices, and to harvest it in a non-enzymatic way. Thus, the integrity of cell surface proteins, ion channels and intercellular connexins is preserved (1). This harvest method markedly improves the utilization of cells and increases the plasticity of the cell sheet. Thus, cell sheets may be implanted directly into the defect site for tissue repair, or multiple homogeneous or heterogeneous cell sheets are constructed by three-dimensional superposition in vitro (2) to repair the defect site. Based on the above-mentioned advantages, the technology has been widely used in tissue and organ repair and regeneration, including myocardial repair, corneal epithelial repair, esophageal endothelial repair and periodontal repair, amongst others (3-5), with certain methods already being applied in clinical practice. In addition, as cell sheet repair does not require scaffold materials, various problems, such as inflammatory response caused by acid production for material degradation, are avoidable.

Gene modification of stem cells refers to the introduction of DNA or RNA into target cells to change their direction of differentiation or secretion of a certain protein, so as to promote the effects of stem cell therapy and tissue regeneration (6). Various micromolecular nucleic acids, including DNA, antisense oligonucleotides, siRNA and miRNA, may all be specifically transfected into cells to exert therapeutic effects on the molecular level (7). Although viral vectors efficiently transfect multiple types of cell, with stable gene expression for a long time, the safety issues concerning clinical immunogenicity and carcinogenicity hinder further development. By contrast, non-viral vectors have many advantages, such as low immune response, easy in vitro synthesis, and modification and safety, so they are preferred to viral vectors in gene therapy. At present, although gene transfection has been widely applied in the gene modification of stem cells, it has seldom been used 
to modify cell sheets. Meanwhile, only certain complicated methods, such as sheet transfection by magnetic force with magnetic bead-modified virus as a vector, have been demonstrated to be effective. Therefore, it is necessary to identify a simple and practical method for transfection if cell sheets are modifiable using genetic engineering technology to facilitate their specific differentiation into a regenerative target.

miRNAs, as a class of non-coding RNAs, inhibit translation through incomplete matching and binding with target mRNA, enabling individual miRNAs to act simultaneously on multiple genes and inhibit their expression (8). Furthermore, each gene is regulated by multiple miRNAs. Accordingly, miRNA is involved in many cell functions, including a variety of metabolic processes, such as proliferation, differentiation and apoptosis (9-11). Different miRNAs promote bone marrow mesenchymal stem cells (BMMSCs) to differentiate in a variety of ways, including osteogenic differentiation. miR-122 is a negative regulatory factor for the osteogenic differentiation of BMMSCs (12), and its inhibitor significantly reduces the level of endogenous miR-122 and improves the osteogenic differentiation capability of stem cells (13).

The aim of the present study was to complex an miR-122-modified BMMSC sheet with a micro-arc titanium oxide implant to construct a gene-modified tissue-engineered implant. By using an unmodified sheet-implant complex as a control, the osteogenic activity and induction ability of the implanted bone were evaluated in vitro and in vivo. The aim of the study was to provide novel ideas and methods for solving clinical problems, including long, poor healing of implanted bones and high failure rate in patients with bone metabolic diseases.

\section{Materials and methods}

Experimental animals and grouping. A total of 18 one-week-old Sprague-Dawley rats (weight, 20-25 g) were obtained from the Experimental Animal Center of First People's Hospital of Qingdao Economic and Technological Development Zone (Qingdao, China), and divided into a control group, an miR-122 control group and an miR-122 group $(n=6)$. The present study was approved by the Ethics Committees on Animal Experimentation at the First People's Hospital of Qingdao Economic and Technological Development Zone and efforts were made to minimize suffering. All rats were fed under specific pathogen-free conditions at $22-25^{\circ} \mathrm{C}$, and given free access to water and food.

Isolation and culture of BMMSCs. The rats were euthanized by cervical dislocation and immersed in $75 \%$ ethanol for $10 \mathrm{~min}$. The femur and tibia were isolated in a culture dish. The contents of the bone marrow cavity were washed out using a 5 -ml syringe and $\alpha$-minimum essential medium ( $\alpha$-MEM) with 15\% fetal bovine serum (FBS) and 1\% penicillin-streptomycin, which were all purchased from Gibco; Thermo Fisher Scientific, Inc. (Waltham, MA, USA), and seeded in a 75-ml culture flask. Subsequently, $\alpha$-MEM was added to $10 \mathrm{ml}$, and routinely cultured in $5 \% \mathrm{CO}_{2}$ and saturated humidity at $37^{\circ} \mathrm{C}$. The medium was refreshed for the first time $72 \mathrm{~h}$ after inoculation to remove non-adherent cells and tissue residues, and the adherent cells were continuously cultured at $37^{\circ} \mathrm{C}$. The medium was refreshed once every two days, and the cells were digested and passaged with $0.25 \%$ trypsin until $70-85 \%$ confluence.

Flow cytometry. First-generation BMMSCs were digested with trypsin, centrifuged at $12,000 \mathrm{x} \mathrm{g}$ and $4^{\circ} \mathrm{C}$ for $5 \mathrm{~min}$ to discard the supernatant, washed twice with phosphate-buffered saline (PBS) containing 3\% FBS, then resuspended with this solution and counted under a light microscope. The cells were subpackaged into eight EP tubes at a density of $1 \times 10^{5}$ cells $/ 200 \mu 1$, and $2 \mu \mathrm{l}$ (1:500) of cluster of differentiation 29 (CD29) (cat. no. 555005), CD90 (cat. no. 554897), CD105 (cat. no. 550546), CD34 (cat. no. 560238), CD45 (cat. no. 553091) and CD31 (cat. no. 555027) antibodies (BD Pharmingen, San Diego, CA, USA) was added into individual tubes. The cells were incubated in the dark at $4^{\circ} \mathrm{C}$ for $1 \mathrm{~h}$, centrifuged at $12,000 \mathrm{x} \mathrm{g}$ and $4^{\circ} \mathrm{C}$ for 5 min to rinse off excess antibodies, resuspended with PBS containing $3 \%$ FBS to $200 \mu 1$ and detected by flow cytometer (BD Biosciences, San Jose, CA, USA).

Adipogenic differentiation. Second-generation BMMSCs were seeded in a six-well culture plate at a density of $3 \times 10^{5}$ cells/well to observe their adherent growth. Upon $>85 \%$ confluence, the medium was replaced with adipogenesis-inducing solution [ $\alpha-$ MEM containing 5\% FBS and $100 \mathrm{nM}$ dexamethasone (Sigma-Aldrich; Merck KGaA, Darmstadt, Germany), 0.5 mM 3-isobutyl-1-methylxanthine, $50 \mathrm{mM}$ indomethacin, $0.01 \mathrm{mg} / \mathrm{ml}$ insulin (Sigma-Aldrich; Merck KGaA), $50 \mu \mathrm{g} / \mathrm{ml}$ ascorbic acid (Sigma-Aldrich; Merck KGaA)] and induced for $\sim 10$ days. The culture medium was refreshed once every 3 days. After lipid droplets formed, the cells were washed three times with PBS, fixed with $4 \%$ paraformaldehyde, stained with Oil Red O for $1 \mathrm{~h}$ at room temperature, observed under an inverted microscope (Olympus, Tokyo, Japan) and photographed.

Osteogenic differentiation. Second-generation BMMSCs were seeded in two six-well culture plates at a density of $3 \times 10^{5}$ cells/well for their adherent growth. Upon confluence of $>85 \%$, the medium was replaced with osteogenesis-inducing solution and refreshed once every 2 days. Alkaline phosphatase (ALP) formation was observed under an inverted microscope by staining using an ALP kit (cat. no. 86C-1KT, Sigma-Aldrich; Merck KGaA). Cells in the other plate were continuously induced until day 28 and stained with $1 \%$ alizarin red for $5 \mathrm{~min}$ at room temperature. Formation of mineralized nodules was observed under an inverted microscope and photographed.

Detection of collagen secretion. The sheet was fixed at each time-point of osteogenic induction, stained with $1 \%$ Sirius Red/picric acid for $1 \mathrm{~h}$ at room temperature (R\&D Systems $\mathrm{GmbH}$, Wiesbaden, Germany) for $18 \mathrm{~h}$, after the excess staining solution was washed away using $0.1 \mathrm{M}$ acetic acid, the sheet was observed under an inverted microscope and photographed. Semi-quantitative analysis of collagen staining was performed as follows: Destaining solution $(1 \mathrm{ml})$ was added into each well (Miltenyi Biotec GmbH, Gladbach, Germany) while shaking for $15 \mathrm{~min}$, and the optical density (OD) was measured at $540 \mathrm{~nm}$ (Thermo Fisher Scientific, Inc.). 
Detection of extracellular matrix mineralization. The sheet was fixed with citrate concentrated solution and acetone at each time-point of osteogenic induction, and $1 \%$ alizarin red (Beyotime Institute of Biotechnology, Haimen, China) was added and stained for $3 \mathrm{~min}$ at room temperature. Subsequent to washing with PBS to remove the excess staining solution, the sheet was observed under an inverted microscope and photographed. Semi-quantitative analysis of mineralized staining was performed as follows: Destaining solution $(1 \mathrm{ml}$; $\mathrm{pH}$ 7.0) was added to each well, while shaking for $15 \mathrm{~min}$, and the optical density was measured at a wavelength of $620 \mathrm{~nm}$ (Thermo Fisher Scientific, Inc.).

Construction of the miR-122-modified BMMSC sheet. First-generation BMMSCs were seeded in six-well culture plates at a density of $3 \times 10^{5}$ cells/well. When the confluence reached $100 \%$, basic culture medium in the plate was replaced with a sheet-inducing medium, and continuously cultured at $37^{\circ} \mathrm{C}$ for 6 days. The culture medium was refreshed once every two days. Liquid in the plate well was replaced with sheet-inducing medium without antibiotics (Sigma-Aldrich; Merck $\mathrm{KGaA}$ ) on day 7 , and a mixture of four types of transfection vectors was added on day 8 to complete the transfection $\left(5 \% \mathrm{CO}_{2}\right.$ and saturated humidity for $24 \mathrm{~h}$ at $\left.<37^{\circ} \mathrm{C}\right)$. The medium was replaced with sheet-inducing medium and continuously incubated at $37^{\circ} \mathrm{C}$ for $24 \mathrm{~h}$ to complete sheet construction.

Scanning electron microscopy (SEM). The obtained sheet was washed with PBS three times, and $3 \%$ glutaraldehyde (pH 7.4) was added and maintained overnight at $4^{\circ} \mathrm{C}$. Gradient dehydration was conducted using 50, 70, 80, 90 and $100 \%$ ethanol solutions. After critical-point drying and gold spraying, the morphology of the sheet was observed under a field emission scanning electron microscope (Hitachi, Ltd., Tokyo, Japan).

Hematoxylin and eosin staining H\&E staining. The sheet harvested from each group was fixed with $4 \%$ paraformaldehyde, conventionally dehydrated, embedded in paraffin, deparaffinized with xylene, soaked in gradient ethanol solutions (3 times, 3 min), deparaffinized by series concentrations of ethanol solutions, stained with hematoxylin at room temperature for $10 \mathrm{~min}$, washed with distilled water, differentiated with $0.5 \%$ hydrochloric acid-ethanol solution for $30 \mathrm{sec}$, colored blue with concentrated aqueous ammonia, washed with distilled water, stained with $0.5 \%$ eosin at room temperature for $3 \mathrm{~min}$ and washed with distilled water. Subsequently, the sheet was routinely dehydrated, transparentized using xylene, sealed with neutral gum, and observed under an optical microscope (Olympus, Tokyo, Japan).

Quantitative polymerase chain reaction $(q P C R)$ detection. All reagents were purchased from Takara Bio, Inc. (Otsu, Japan) and the cell sheets were washed three times with PBS. Cells $\left(10^{6}\right)$ were directly added to $1 \mathrm{ml}$ TRIzol, mixed in a vortex oscillator, left still at room temperature for $5 \mathrm{~min}$, mixed with $1 / 5$ volume of chloroform for $1 \mathrm{~min}$, left still at room temperature for $5 \mathrm{~min}$, centrifuged at $4^{\circ} \mathrm{C}$ and $12,000 \mathrm{x}$ g for $15 \mathrm{~min}$, mixed with an equal volume of isopropanol, left still at room temperature for $10 \mathrm{~min}$ and centrifuged again at $4^{\circ} \mathrm{C}$ and $12,000 \mathrm{x} \mathrm{g}$ for $10 \mathrm{~min}$. After the supernatant was discarded, $1 \mathrm{ml}$ of $75 \%$ ethanol was added, and an appropriate quantity of diethylpyrocarbonate-water was added to completely dissolve the precipitate. The $25-\mu 1$ reaction system contained the following: Fluorescent reverse transcription (RT)-PCR reaction solution (20 $\mu \mathrm{l}), 1 \mu \mathrm{l}$ DNA polymerase, $0.35 \mu 1$ reverse transcriptase and $5 \mu$ l template RNA. The mixture was centrifuged at $6,000 \mathrm{x}$ g for $10 \mathrm{sec}$. The reaction conditions for miRNA RT were as follows: One cycle of pre-denaturation at $95^{\circ} \mathrm{C}$ for $30 \mathrm{sec}$. The PCR reaction was as follows: Forty cycles at $95^{\circ} \mathrm{C}$ for $5 \mathrm{sec}$, at $55^{\circ} \mathrm{C}$ for $30 \mathrm{sec}$ and at $72^{\circ} \mathrm{C}$ for $30 \mathrm{sec}$, and melting curve analysis was conducted at $95^{\circ} \mathrm{C}$ for $5 \mathrm{sec}$. The procedure for quantitative fluorescent RT-PCR was as follows: RT at $50^{\circ} \mathrm{C}$ for $30 \mathrm{~min}$, pre-denaturation at $95^{\circ} \mathrm{C}$ for $3 \mathrm{~min}$, denaturation at $95^{\circ} \mathrm{C}$ for $15 \mathrm{sec}$, annealing at $50^{\circ} \mathrm{C}$ for $30 \mathrm{sec}$ and extension at $72^{\circ} \mathrm{C}$ for $30 \mathrm{~min}$ (5 cycles in total); denaturation at $95^{\circ} \mathrm{C}$ for $10 \mathrm{sec}$ and annealing at $55^{\circ} \mathrm{C}$ for $40 \mathrm{sec}(40$ cycles in total). The relative expression level was calculated according to $\mathrm{X}=2^{-\Delta \Delta \mathrm{Cq}}$ with U6 serving as the internal reference (14). Primer sequences for the PCR system (Roche Innovatis, Bielefeld, Germany) were as follows. Upstream, 5'-CCACTACGGTCTTCACAG GACCT-3' and downstream, 5'-TCTTCAGTTGCCTTCTTG GTTC-3' for runt-related transcription factor 2 (RUNX2); upstream, 5'-AAGGCAGTTGGCAGTAGTGG-3' and downstream, 5'-TGAATGGGCTTCTTCCTCAG-3' for osterix (OSX); upstream, 5'-AACGTGGCCAAGAACATCATCA-3' and downstream, 5'-TGTCCATCTCCA ACCTGCAC-3' for ALP; upstream, 5'-GCCTCCCAGAACATCACCTA-3' and downstream, 5'-AGTCGTGTCGATCCGTAGC-3' for collagen I (COL-I); upstream, 5'-CATGGTGCTAGCTAG AGCTTCC-3' and downstream, 5'-TTCCTGACGTGATCG AAGTTCC-3' for bone morphogenetic protein 2 (BMP-2); upstream, 5'-GGTGCAGACCTAGCAGACACCA-3' and downstream, 5'-AGGTAGCGCCGGAGTCTATTCA-3' for osteocalcin (OCN); upstream, 5'-GGCACAGTCAAGGCT GAGAATG-3' and downstream, 5'-ATGGTCATGCAAGAC GCCAGTA-3' for GAPDH:

Western blot analysis. Sheet protein ( $1 \mathrm{mg}$, quantified by the bicinchoninic acid method) from each group was subjected to $12 \%$ SDS-PAGE. Then two pieces of gel were carefully peeled off, and sequentially paved sponge pad, filter paper, gel, sheet, filter paper and sponge pad to prepare a gel transfer interlayer from which bubbles were removed using a glass rod. Subsequently, it was placed into an electrical slot and transferred for $90 \mathrm{~min}$ at $90 \mathrm{~V}(330 \mathrm{~mA})$. The membrane was transferred to a plate containing $25 \mathrm{ml}$ blocking solution using forceps, shaken slowly for $1 \mathrm{~h}$ at room temperature, and ALP (cat. no. ab67228), COL-1 (cat. no. ab34710), RUNX2 (cat. no. ab76956), OSX (cat. no. ab22552), OCN (cat. no. ab13420), extracellular signal-regulated kinases (ERKs) 1/2 (cat. no. ab17942) and phosphorylated (p)-ERK 1/2 (cat. no. ab201015) antibodies (all 1:1,000 diluted; Abcam, Cambridge, UK) were added and incubated overnight at $4^{\circ} \mathrm{C}$. The membrane was washed with Tris-buffered saline and Tween-20 (TBST) three times ( 5 min each time) at room temperature in a shaker and the next day horseradish peroxidase-conjugated secondary antibody (1:1,000 diluted, cat. no. BA1058; Wuhan Boster Biological Technology, Ltd., 

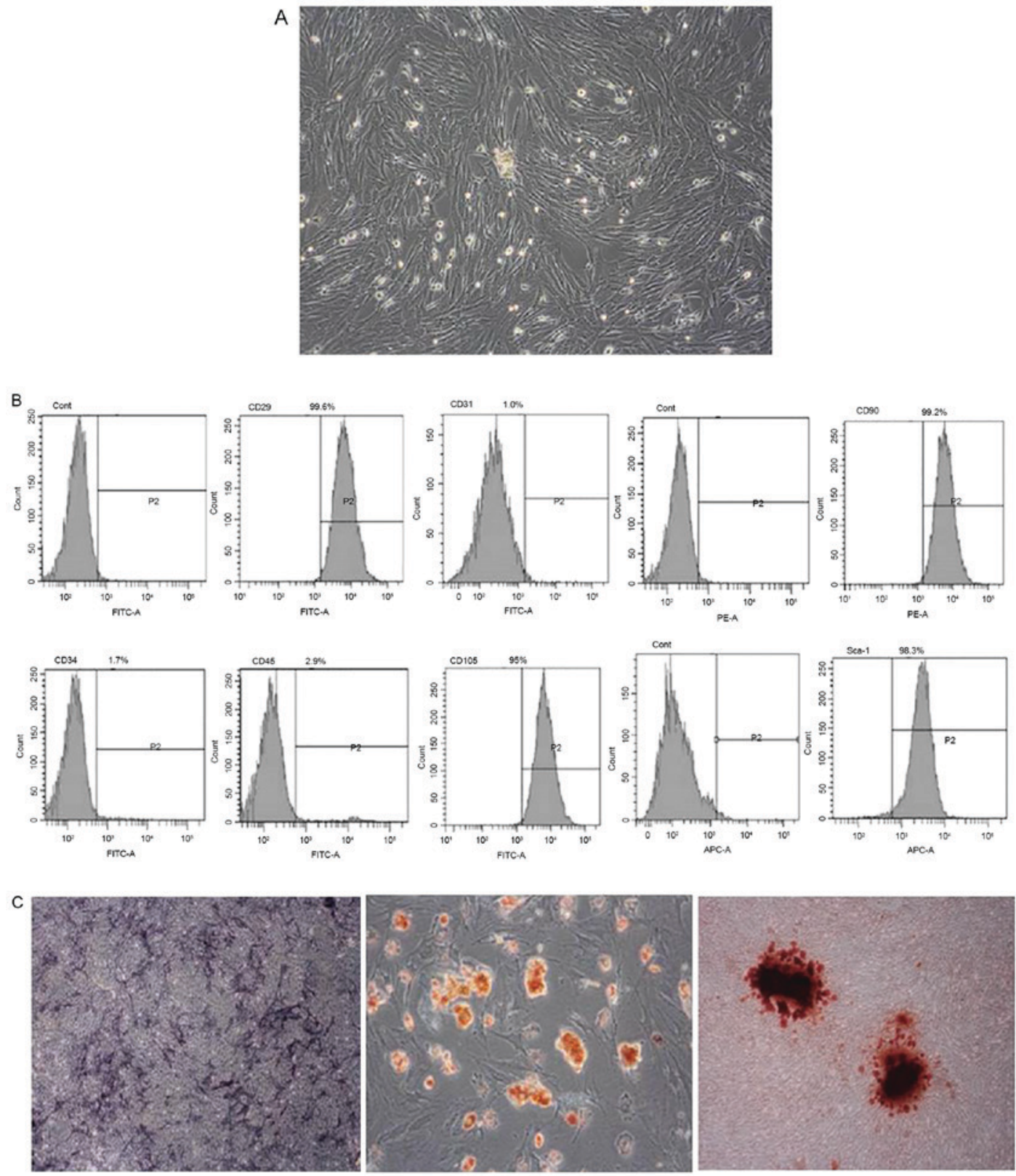

Figure 1. Isolation, culture and identification of BMMSCs. (A) Rat BMMSCs (magnification, x400). (B) Flow cytometry results of cell surface markers (C) left, alkaline phosphatase staining results after 7 days of osteogenic induction; middle, Oil Red O staining results after 10 days of adipogenic induction; right, alizarin red staining results after three weeks of osteogenic induction (magnification, x400). BMMSCs, bone marrow mesenchymal stem cells; $\mathrm{CD}$, cluster of differentiation; Cont, control; FITC, fluorescein isothiocyanate; PE, phycoerythrin; APC, allophycocyanin.

Wuhan, China) was added, incubated in a shaker at $37^{\circ} \mathrm{C}$ for $1 \mathrm{~h}$, washed with TBST three times (5 min each time) and color-developed by diaminobenzidine solution (Thermo Fisher Scientific, Inc.).

Immunohistochemical staining. Seven days after osteogenic induction, cell sheets of the three groups were washed with PBS, fixed with $4 \%$ paraformaldehyde for $24 \mathrm{~h}$, dehydrated using a dehydrator, embedded in paraffin, serially sectioned $(5 \mu \mathrm{m})$ using a slicing machine, and deparaffinized with gradient xylene and ethanol solutions to water. After a paraffin circle was made, the sheets were repaired with $1 \%$ trypsin at $37^{\circ} \mathrm{C}$ for $30 \mathrm{~min}$, washed with PBS three times $(5 \mathrm{~min}$ each time), treated with $3 \%$ hydrogen peroxide for $15 \mathrm{~min}$, washed with PBS three times (5 min each time) and blocked with goat serum (Sigma-Aldrich; Merck KGaA) at $37^{\circ} \mathrm{C}$ for $1 \mathrm{~h}$. Suitable concentrations of ALP, COL-1, OCN and RUNX2 primary antibodies (1:150; Cell Signaling Technology, Inc., Danvers, MA, USA) were added and incubated at $4^{\circ} \mathrm{C}$ overnight, rinsed with PBS three times (5 min each time), and universal secondary antibody (1:50) (Biosynthesis, Beijing, China) was added and incubated at $37^{\circ} \mathrm{C}$ for $2 \mathrm{~h}$, washed with PBS three times (5 min each time), stained with 3,3-diaminobenzidine color-developing solution for $15 \mathrm{~min}$ at room temperature, observed under a microscope and photographed.

Preparation of micro-arc titanium oxide implant. Pure titanium samples were polished with silicon carbide abrasive papers (Agilent Technologies, Inc., Santa Clara, CA, USA) from 400 to 1,000 meshes sequentially, ultrasonically cleaned 

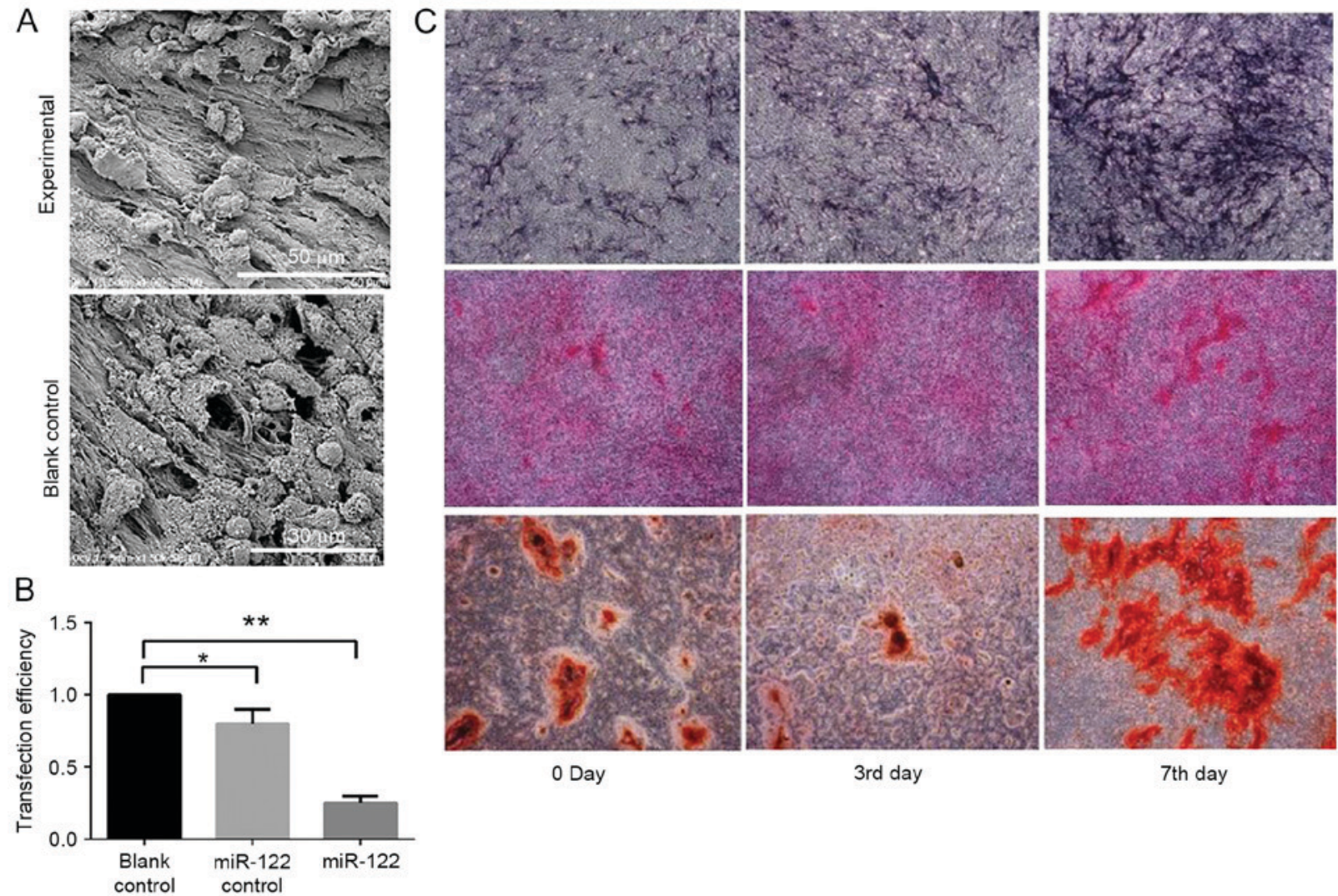

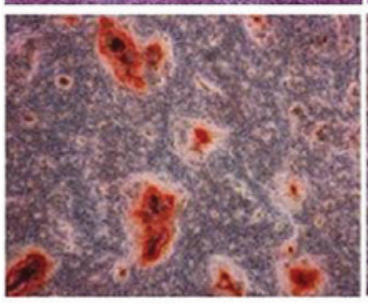

0 Day

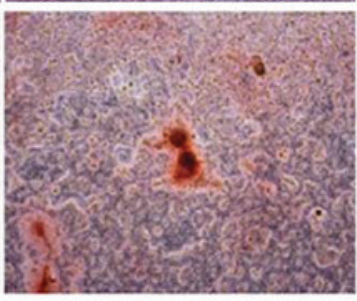

3rd day

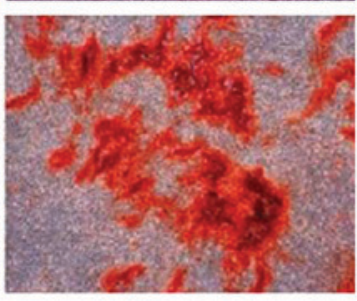

7 th day

Figure 2. Morphology of miR-122-modified sheet. (A) Scanning electron microscopy images demonstrated that miR-122-modified and unmodified sheets were rich in intracellular and extracellular matrices. (B) Dose-dependence of miR-122 transfection. (C) Alkaline phosphatase (top), Sirius Red (middle) and extracellular matrix (bottom) staining indicated that the experimental group had significantly higher osteogenic capability and collagen secretion than those of the two control groups (magnification, $\mathrm{x} 400$ ). ${ }^{*} \mathrm{P}<0.05$ and ${ }^{* *} \mathrm{P}<0.01$ vs. blank control group.

in deioni zed water (Thermo Fisher Scientific, Inc.) and dried in air at room temperature. A micro-arc oxidation electrolyte solution (GE Healthcare Life Sciences, Little Chalfont, UK) was formulated with $0.02 \mathrm{M} \beta$-glycerol phosphate and $0.2 \mathrm{M}$ citric acid. The samples were subjected to micro-arc oxidation treatment for $5 \mathrm{~min}$ at $400 \mathrm{~V}, 100 \mathrm{~Hz}$ and $20 \%$ duty ratio. Subsequently, ultrasonic cleaning was conducted successively in acetone, absolute ethanol and deionized water for $10 \mathrm{~min}$ each, and dried in air at room temperature. Finally, the samples were sealed and packaged for Co60 (Thermo Fisher Scientific, Inc.) irradiation sterilization.

Statistical analysis. All data were expressed as means \pm standard deviation and analyzed using SPSS 16.0 (SPSS, Inc., Chicago, IL, USA). Groups were compared by one-way analysis of variance, and pairwise comparison was performed using Tukey's post hoc test. $\mathrm{P}<0.05$ was considered to indicate a statistically significant difference.

\section{Results}

Isolation, culture and identification of BMMSCs. Primary BMMSCs were cultured using the whole bone marrow adherence method, which almost reached complete confluence 7 days after inoculation. The cells were mostly spindle- or triangle-shaped, with large nuclei and high refractive indices. Flow cytometry demonstrated that surface markers, CD29 (99.6\%), CD90 (99.2\%) and CD105 (95\%) of BMMSCs were positively expressed, whereas blood cell markers, CD34 (1.7\%), CD45 (2.9\%) and CD31 (1.0\%) were negatively expressed. The results conformed to the characteristics of mesenchyme-derived stem cells. After 10 days of adipogenic induction, Oil Red O staining exhibited the scattered distribution of red lipid droplets in and between the cells. ALP staining had positive results after 7 days of osteogenic induction, showing bluish purple intracellular crystals. Calcified nodules formed on the cell surface after three weeks of induction, as indicated by alizarin red staining (Fig. 1).

Morphology of miR-122-modified sheet. SEM images demonstrated that miR-122-modified and unmodified sheets were rich in intracellular and extracellular matrices. Flow cytometry identified that miR-122 transfection was dose-dependent. In addition, ALP staining was conducted after 3 and 7 days of osteogenic induction. ALP expression levels in the miR-122-modified sheet exceeded those in the blank control and negative control groups. Furthermore, Sirius Red staining and semi-quantitative analysis revealed that the experimental group secreted significantly more collagen than the control groups (Fig. 2).

Expression levels of osteogenesis-associated functional genes and proteins. RUNX2 and OSX are osteogenesis-associated genes. ALP and COL-1 are early osteogenic markers, while OCN and BMP-2 are late ones. On day 3 of osteogenic induction, the RUNX2, OSX, OCN, COL-1, ALP and BMP-2 
A
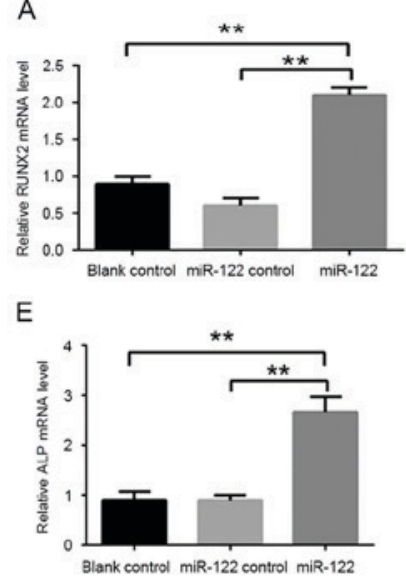

B

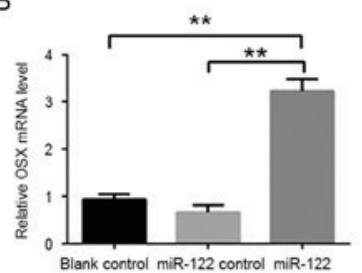

C

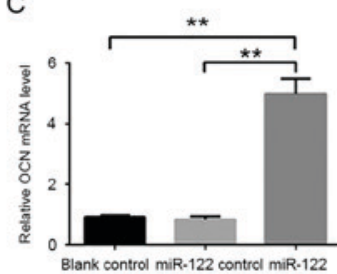

D

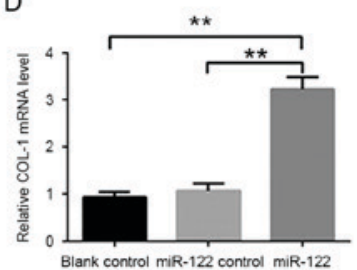

$\mathrm{F}$

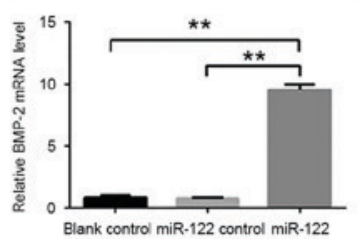

$\mathrm{H}$

G

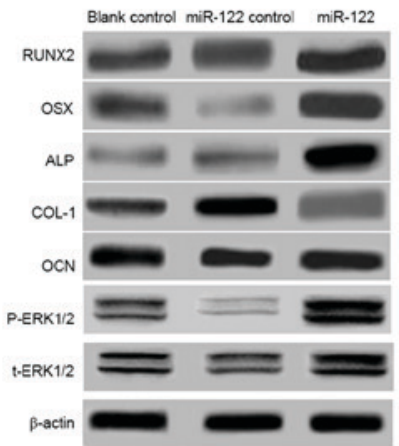

Figure 3. Expression levels of osteogenesis-associated functional genes and proteins. (A-F) Quantitative polymerase chain reaction results for RUNX2, OSX, ALP, COL-1, OCN and BMP-2 after three days of osteogenic induction. (G) Expression levels of osteogenesis-associated proteins (RUNX2, OSX, ALP and OCN) and t-ERK 1/2, p-ERK $1 / 2$ and ERK 1/2 signaling pathways after three days of osteogenic induction. (H) Immunohistochemical assay results of COL-1, ALP, OCN and RUNX2 proteins after three days of osteogenic induction. ${ }^{* *} \mathrm{P}<0.01$ vs. miR-122 group. RUNX2, runt-related transcription factor 2; OSX, osterix; ALP, alkaline phosphatase; COL-1, collagen I; OCN, osteocalcin; BMP-2, bone morphogenetic protein 2; OSX, osterix; ERK, extracellular signal-regulated kinases; t, total; p, phosphorylated.

expression levels of the experimental group were 2.0, 3.1, 4.6, 3.2, 10.5 and 4.5 times those of the blank control group, respectively. Western blotting and immunohistochemical assay demonstrated that significantly more RUNX2, OSX, ALP and $\mathrm{OCN}$, as well as significantly less COL-1, were expressed in the experimental group when compared with those of the two control groups $(\mathrm{P}<0.05)$. As evidenced by western blotting, the p-ERK $1 / 2$ expression level was only upregulated in the experimental group following miR-122 transfection (Fig. 3).

Underlying mechanism for osteogenic differentiation of BMMSC sheet promoted by miR-122. SEM was performed $48 \mathrm{~h}$ after complexation between the miR-122-transfected cell sheet and implant. Numerous collagenous fibers and cells containing abundant extracellular matrices were interlinked into a layer of network structure that closely adhered to the porous surface of the implant. Under high magnification, the cells were extended, with a large quantity of pseudopodia protruding toward and fitting the extracellular matrices. On day 3 of osteogenic induction after complexation of the sheet and implant, the BMP-2, RUNX2, ALP, OSX and COL-1 expression levels of the experimental group were 1.2, 1.6,
2.2, 2.1, 1.3 and 3.3 times those of the blank control group, respectively. In the 4th week, large-area, paralleling bone-like mineralized tissues formed near the implant of the experimental group, in which there were considerable bone lacunae and osteocytes containing new blood vessels. By contrast, the implants of the two control groups were primarily subjected to fibrous repair, only accompanied by formation of a small quantity of bones. In the 8th week, there were widely distributed, dense and well mineralized mature bone tissues on the implant surface of the experimental group, which formed good osseointegration with the implant. The new bones had typical lacunae, osteocytes and bone lining cells, which were accompanied by angiogenesis. In addition, the bones predominantly formed through endochondral ossification. As to the implants of the two control groups, significantly fewer bones formed and the degree of mineralization was low (Fig. 4).

\section{Discussion}

In the present study, BMMSCs were isolated and cultured using the whole bone marrow adherence method, as fibroblast-like cells with clonal or whirlpool growth patterns. Flow 
A

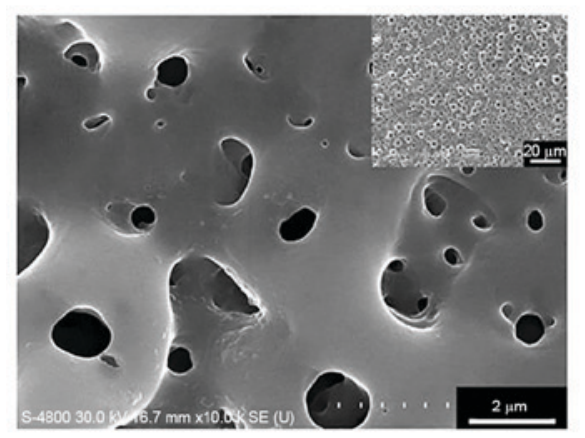

C
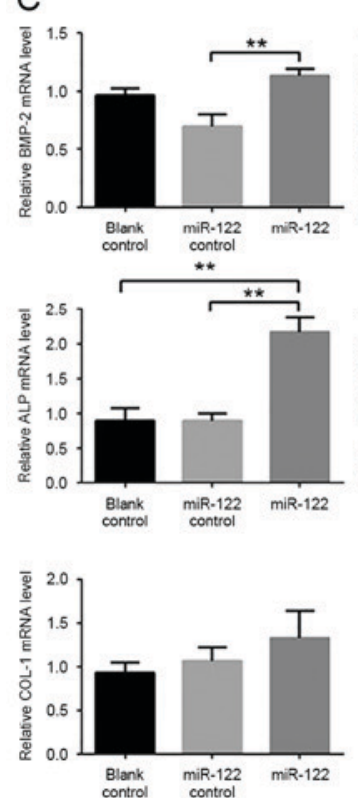
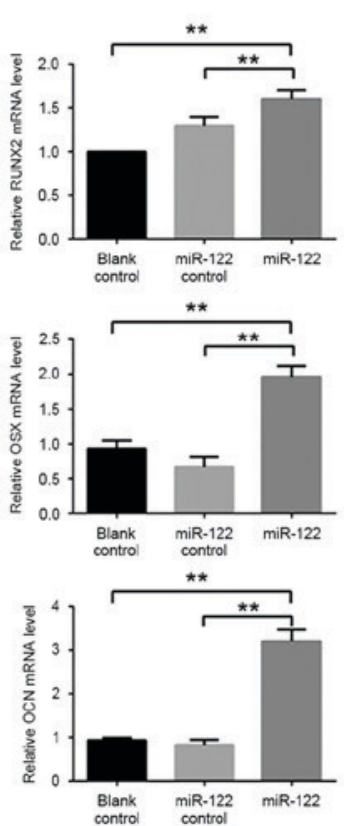

B

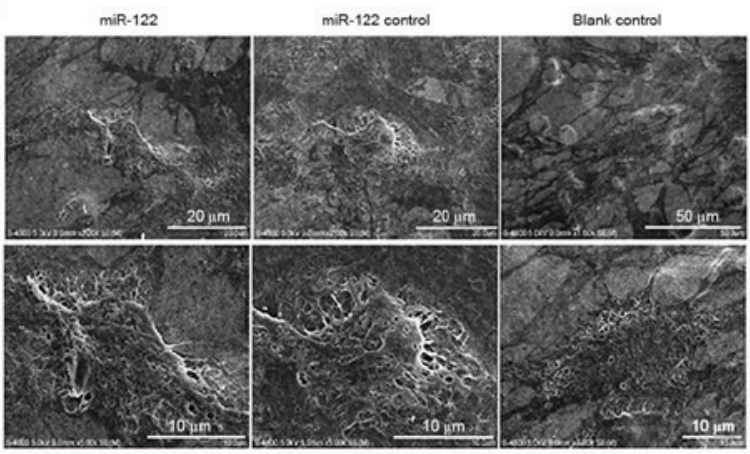

D
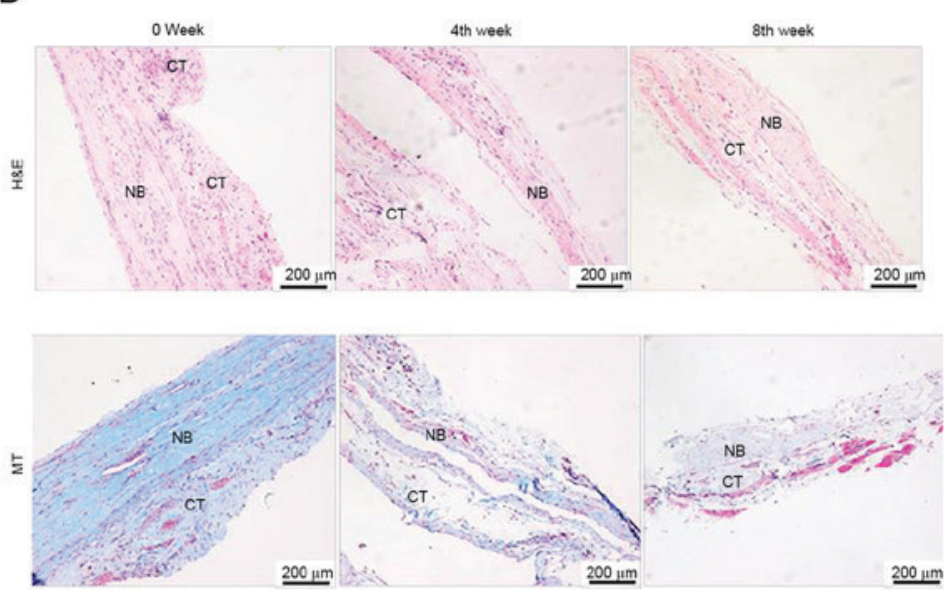

Figure 4. Underlying mechanism for osteogenic differentiation of the bone marrow mesenchymal stem cells sheet promoted by miR-122. (A and B) Scanning electron microscopy was performed $48 \mathrm{~h}$ after complexation between the miR-122-transfected cell sheet and implant. (C) Quantitative polymerase chain reaction results of BMP-2, RUNX2, ALP, OSX and COL-1 expression levels after 3 days of osteogenic induction. (D) Histological observation of implants after 4 and 8 weeks of induction using hematoxylin and eosin and Masson's trichrome ${ }^{* *} \mathrm{P}<0.01$ vs. miR-122 group. BMP-2, bone morphogenetic protein 2; RUNX2, runt-related transcription factor 2; ALP, alkaline phosphatase; OSX, osterix; COL-1, collagen I; OCN, osteocalcin; OSX, osterix.

cytometry indicated that surface markers, CD29, CD90 and CD105 were positively expressed on the surface of BMMSCs, although blood cell markers, CD34, CD45 and CD31 were negatively expressed. Therefore, the obtained BMMSCs were mesenchyme-derived cells.

Multi-lineage differentiation potential in vitro is one of the important features of stem cells (15), which was detected in the present study by in vitro adipogenic and osteogenic induction tests. As ALP staining was strongly positive after 7 days of osteogenic induction, BMMSCS began to transform into osteoblasts and thereafter became typical ones, as indicated by the strongly positive staining of calcified nodules 14 days later. Thus, the obtained BMMSCs were able to differentiate into osteoblasts. In the meantime, after 10 days of adipogenic induction, the cells transformed from spindle- to polygonal-shaped, and lipid droplets (similar in appearance to a string of beads) were observed in the cytoplasm and between the cells. Given that Oil Red O staining showed positive results, BMMSCs could differentiate into adipocytes.

RNA interference refers to specific binding of exogenous or endogenous siRNA or miRNA to corresponding target gene mRNA after entering cells, thereby inhibiting protein translation $(16,17)$ and expressions of specific genes. Typically, siRNA leads to complete silencing of specific target genes, while miRNA acts on multiple genes and inhibits their expression levels (18). Additionally, miRNA regulates numerous metabolic processes, such as cell proliferation, differentiation and apoptosis, managing to promote different directions of differentiation of MSCs (including osteogenic differentiation of stem cells) (19-22). In the present study, the osteogenic capability of miR-122-transfected BMMSC sheet was comprehensively evaluated by ALP staining, analyses of collagen secretion and extracellular matrix mineralization, as well as determination of osteogenesis-associated gene and protein expression levels. Based on the osteogenic parameters after 3 and 7 days of induction, miR-122 transfection significantly promoted the osteogenic differentiation of BMMSC sheets.

It is well-documented that miRNA exerts key regulatory effects on the proliferation and differentiation of stem cells (23), although the detailed molecular mechanisms remain elusive. Furthermore, in the present study, endogenous miR-122 
expression levels were detected in the sheets of different groups at various stages of osteogenic induction using PCR. miR-122 modification continuously inhibited such expression throughout the process. ERK $1 / 2$ is a regulatory kinase for the mitogen-activated protein kinases (MAPK) signaling pathway. Notably, p-ERK $1 / 2$ is involved in various biological processes of osteoblasts, such as proliferation, differentiation and apoptosis (24).

In the present study, the expression levels of p-ERK 1/2, RUNX2 and osteogenesis-associated genes in the cell sheet of an experimental group were detected. As a key transcription factor for the osteogenic differentiation of BMMSCs, RUNX2 directly regulates the transcription of OCN, COL-I and osteopontin, with its activity regulated by an MAPK-dependent phosphorylation cascade and activated by ERK $1 / 2$. Furthermore, miR-122 suppresses the osteogenic differentiation of human BMMSCs by inhibiting the ERK 1/2 signaling pathway (25).

In conclusion, it was verified that genetically modifying BMMSC sheets with a non-viral vector is feasible. The resulting tissue-engineered implant provides novel strategies for circumventing long, poor healing implanted bones and their high failure rate. Further studies using more animals and human subjects should be performed to validate the results of the present study.

\section{References}

1. Masuda S and Shimizu T: Three-dimensional cardiac tissue fabrication based on cell sheet technology. Adv Drug Deliv Rev 96: 103-109, 2016.

2. Clark AG and Vignjevic DM: Modes of cancer cell invasion and the role of the microenvironment. Curr Opin Cell Biol 36: 13-22, 2015.

3. Chian KS, Leong MF and Kono K: Regenerative medicine for oesophageal reconstruction after cancer treatment. Lancet Oncol 16: e84-e92, 2015.

4. Westrate LM, Lee JE, Prinz WA and Voeltz GK: Form follows function: The importance of endoplasmic reticulum shape. Annu Rev Biochem 84: 791-811, 2015.

5. Pocha SM and Montell DJ: Cellular and molecular mechanisms of single and collective cell migrations in Drosophila: Themes and variations. Annu Rev Genet 48: 295-318, 2014.

6. Sugimura R: Bioengineering hematopoietic stem cell niche toward regenerative medicine. Adv Drug Deliv Rev 99: 212-220, 2016.

7. Flor TB and Blom B: Pathogens use and abuse microRNAs to deceive the immune system. Int J Mol Sci 17: 538, 2016.

8. Hayes CN and Chayama K: MicroRNAs as biomarkers for liver disease and hepatocellular carcinoma. Int J Mol Sci 17: 280, 2016
9. Huang JT, Liu SM, Ma H, Yang Y, Zhang X, Sun H, Zhang X, Xu J and Wang J: Systematic review and meta-analysis: Circulating miRNAs for diagnosis of hepatocellular carcinoma. J Cell Physiol 231: 328-335, 2016.

10. Farooqi AA, Fayyaz S, Shatynska-Mytsyk I, Javed Z, Jabeen S, Yaylim I, Gasparri ML and Panici PB: Is miR-34a a well-equipped swordsman to conquer temple of molecular oncology? Chem Biol Drug Des 87: 321-334, 2016.

11. Chmielewska AM, Rychłowska M, Król E, Solarz K and Bieńkowska-Szewczyk K: Novel methods of hepatitis C treatment and prevention. Postepy Hig Med Dosw (Online) 69: 946-963, 2015.

12. Stachowiak G, Zajac A, Nowak M, Stetkiewicz T and Wilczyński JR: Hemostatic disorders of the menopausal period: The role of microRNA. Prz Menopauzalny 14: 144-148, 2015.

13. Casas-Agustench P, Iglesias-Gutiérrez E and Dávalos A: Mother's nutritional miRNA legacy: Nutrition during pregnancy and its possible implications to develop cardiometabolic disease in later life. Pharmacol Res 100: 322-334, 2015.

14. Livak KJ and Schmittgen TD: Analysis of relative gene expression data using real-time quantitative PCR and the 2(-Delta Delta C(T)) method. Methods 25: 402-408, 2001.

15. Ivanovska IL, Shin JW, Swift J and Discher DE: Stem cell mechanobiology: Diverse lessons from bone marrow. Trends Cell Biol 25: 523-532, 2015.

16. Willeit P, Skroblin P, Kiechl S, Fernández-Hernando $\mathrm{C}$ and Mayr M: Liver microRNAs: Potential mediators and biomarkers for metabolic and cardiovascular disease? Eur Heart J 37: 3260-3266, 2016.

17. McGill MR and Jaeschke H: MicroRNAs as signaling mediators and biomarkers of drug- and chemical-induced liver injury. J Clin Med 4: 1063-1078, 2015.

18. Sedano CD and Sarnow P: Interaction of host cell microRNAs with the HCV RNA genome during infection of liver cells. Semin Liver Dis 35: 75-80, 2015.

19. Motavaf M, Safari S and Alavian SM: Targeting microRNA-122: Walking on cutting edge of hepatitis $\mathrm{C}$ virus infection therapy. Acta Virol 58: 301-308, 2014.

20. He L, Tian DA, Li PY and He XX: Mouse models of liver cancer: Progress and recommendations. Oncotarget 6: 23306-23322, 2015.

21. Gibson NW: Engineered microRNA therapeutics. J R Coll Physicians Edinb 44: 196-200, 2014.

22. Wilson JA and Sagan SM: Hepatitis C virus and human miR-122: Insights from the bench to the clinic. Curr Opin Virol 7: 11-18, 2014.

23. Gupta P, Cairns MJ and Saksena NK: Regulation of gene expression by microRNA in HCV infection and HCV-mediated hepatocellular carcinoma. Virol J 11: 64, 2014.

24. Baek J, Kang S and Min H: MicroRNA-targeting therapeutics for hepatitis C. Arch Pharm Res 37: 299-305, 2014.

25. Qiu Z and Dai Y: Roadmap of miR-122-related clinical application from bench to bedside. Expert Opin Investig Drugs 23: 347-355, 2014.

This work is licensed under a Creative Commons Attribution-NonCommercial-NoDerivatives 4.0 International (CC BY-NC-ND 4.0) License. 